\title{
MBE growth and structural properties of InAs and InGaAs nanowires with different mole fraction of In on Si and strongly mismatched $\mathrm{SiC} / \mathrm{Si}(111)$ substrates
}

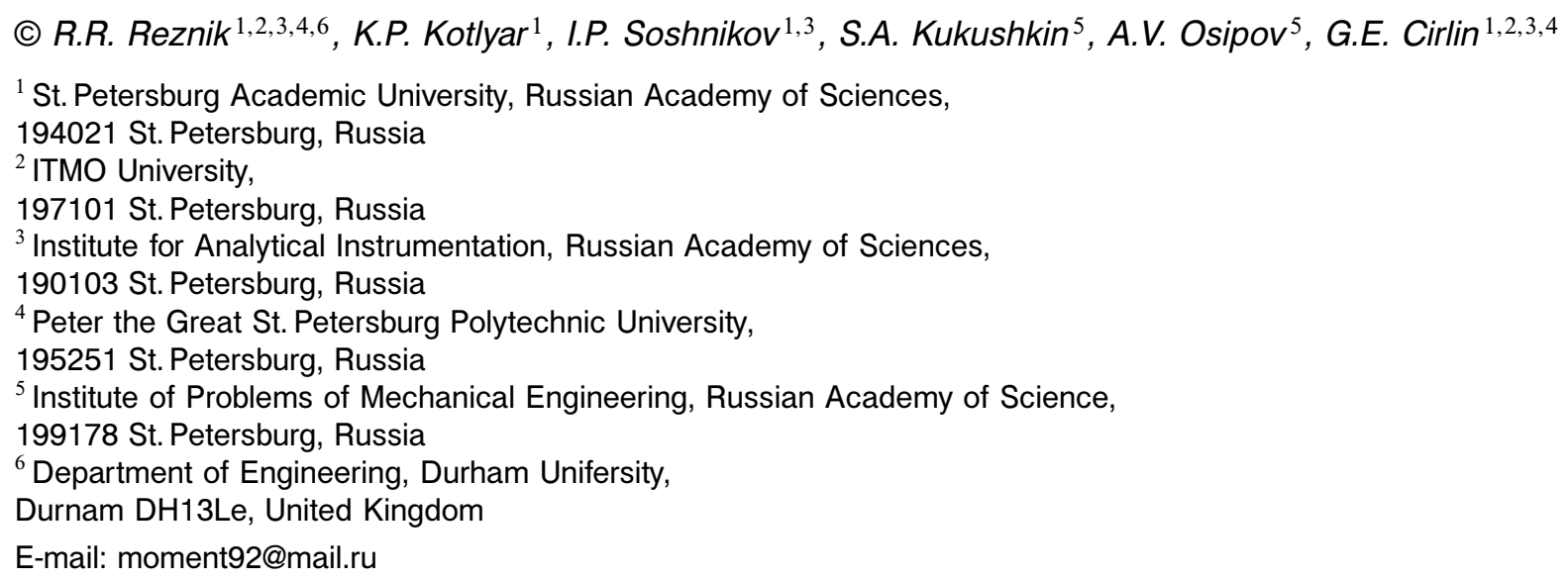

The possibility of InAs nanowires MBE growth on silicon (111) substrates with a nanometer buffer layer of silicon carbide has been demonstrated for the first time. The NWs diameter turned out to be smaller than on the silicon substrate - the minimum of NWs diameter was less than $10 \mathrm{~nm}$. In addition, dependence of structural properties of InGaAs nanowires on composition was studied.

\section{Acknowledgements}

We are grateful for the support of the Ministry of education and science of Russian Federation (state task, project No 16.2483.2017/4.6). The nanowire samples were grown under the support of Russian Science Foundation (Project No 14-12-00393). This work was partially supported by RFBR (grant 16-29-03113 ofi_m). 